\title{
Prioritizing selection of new elements: Bottom-up versus top-down control
}

\author{
MIEKE DONK and JAN THEEUWES \\ Vrije Universiteit, Amsterdam, The Netherlands
}

\begin{abstract}
Watson and Humphreys (1997) have proposed that prioritized selection of new over old elements occurs because observers can apply top-down inhibition to the locations of the old elements by a mechanism they refer to as visual marking. However, recent evidence has suggested that the top-down mechanism is questionable (Donk \& Theeuwes, 2001). In the present study, we investigated whether prioritized selection of new over old elements occurs in a bottom-up or a top-down fashion. Observers were presented with displays containing one set of elements (old elements) followed, after a certain time interval, by a second set of elements (new elements). The observers were instructed to search for the presence of a target that was presented with equal probability among the old and the new elements (Experiments 1 and 2) or twice as often among the old elements than among the new elements (Experiment 3). The results show that new elements were prioritized for selection over old ones even though the observers had no incentive to do so. The results suggest that prioritized selection of new over old elements is not mediated by a top-down inhibition process, as was proposed by Watson and Humphreys (1997). Instead, prioritization of new elements appears to be a bottom-up process. The implications of these results are discussed in terms of models of attentional control.
\end{abstract}

In everyday life, the human observer is continuously confronted with an enormous influx of visual information. Yet the human information-processing capacity is limited (e.g., Allport, 1987; Broadbent, 1958). To behave efficiently, the visual system must select only that information that is relevant to behavioral goals. That is, the visual system must attend to relevant information and ignore irrelevant stimuli. A major issue within the area of visual attention research is the question of how the visual system prioritizes relevant information over irrelevant information. What information can be used to guide attention, and what is the mechanism underlying attentional selection?

Recently, Watson and Humphreys (1997) demonstrated that the human visual system is able to prioritize the selection of multiple new events at the expense of events that have already occurred. They used a paradigm in which they employed a variant of a standard color-form conjunction task. In this paradigm, referred to as the preview paradigm, they compared performance in a single-feature condition (search for a blue $H$ among blue $A$ s) and a conjunction condition (search for a blue $H$ among green $H \mathrm{~s}$ and blue $A$ s) with that in a so-called preview condition, which was identical to the conjunction condition except that one set of elements (the green $H$ s) was presented

We thank Chip Folk, Derrick Watson, Jeremy Wolfe, and one anonymous reviewer for their helpful comments on earlier versions of this article. Correspondence concerning this article should be addressed to M. Donk, Department of Cognitive Psychology, Vrije Universiteit, van der Boechorststraat 1, 1081 BT Amsterdam, The Netherlands (e-mail: w.donk@psy.vu.nl).
$1,000 \mathrm{msec}$ prior to the appearance of the other set of elements (the blue $A \mathrm{~s}$ and, in the target-present trials, the blue $H$ ). The major result consisted of a preview benefit showing that search efficiency in the preview condition was much better than that obtained in the conjunction condition, in which all elements were presented simultaneously. In fact, search efficiency in the preview condition was similar to that obtained in the single-feature condition. Permitting observers a preview of the green elements allowed them to prioritize the new blue elements for selection over the old green ones. To account for the preview benefit, Watson and Humphreys (1997) proposed that the visual system is able to prioritize new information because observers may choose to actively inhibit the locations or other properties of old objects so that these objects no longer compete as strongly for selection. They argued that this inhibition process, denoted as visual marking, is a resource-limited intentional topdown process that can be voluntarily switched on and off (Watson \& Humphreys, 1997).

Even though the preview benefit convincingly demonstrates that observers have the ability to prioritize new over old elements (but see Theeuwes, Kramer, \& Atchley, 1998), it is unclear whether this ability is indeed the result of a top-down goal-driven process. Instead, it is possible that prioritizing occurs because new elements automatically attract attention in a bottom-up fashion. Indeed, recently, Donk and Theeuwes (2001) showed that prioritizing selection of multiple new elements is critically dependent on whether or not the appearance of new elements is accompanied by an abrupt onset. ${ }^{1}$ If new elements were presented with abrupt onsets, search per- 
formance was independent of the number of old elements, showing that the new elements were prioritized. However, if there was no onset of new elements, search performance depended on both the number of new elements and the number of old elements. In other words, for the preview benefit to occur, new elements are required to appear with luminance onset. This suggests that prioritized selection of new elements might be mediated by a bottom-up process that is based on attentional capture by the onsets accompanying the appearance of the new elements (e.g., Yantis \& Hillstrom, 1994; Yantis \& Johnson, 1990; Yantis \& Jones, 1991; Yantis \& Jonides, 1984) and not, as was proposed by Watson and Humphreys (1997), by a top-down process.

Since the introduction of the visual-marking account (Watson \& Humphreys, 1997), there have been several studies performed to support the view that prioritized selection of new over old elements is mediated by topdown processing (Olivers \& Humphreys, 2002; Watson $\&$ Humphreys, 1997, 2000). However, the results of these studies do not provide unequivocal evidence for a top-down account. In fact, the results are also in line with a bottom-up view.

In one study, Watson and Humphreys (1997, Experiment 8) had participants perform a secondary central load task during the preview period when only the old elements were presented. The central load task consisted of naming aloud a series of numbers that were rapidly presented at central fixation. The results demonstrated that with a secondary central load task, search efficiency in the preview condition was less than that obtained without a central load task. According to Watson and Humphreys (1997), because resources were allocated to the central load task, the participants had less spare capacity to actively inhibit the old elements. Since the allocation of resources could be flexibly withheld from the search task, they concluded that the mechanism allowing prioritized selection of new elements is under top-down control. Even though these results are indeed in line with a topdown account, there is yet another explanation. Watson and Humphreys employed an attention-demanding central task that necessitated focus of attention on fixation by the participants. Typically, when participants engage in a focused attentional state, the capture of attention by onsets elsewhere is prevented (Theeuwes, 1994; Yantis $\&$ Jonides, 1990). Consequently, the lower search efficiency in the preview condition with the load task might have been the result of spatial focusing, preventing the new elements from capturing attention. Accordingly, the results of this study do not provide clear evidence for a top-down account.

In another study Watson and Humphreys (2000) addressed the issue of attentional control by utilizing a probe detection task (see also Olivers \& Humphreys, 2002). In this study, they had participants perform a preview search task while, on a minority of the trials, a tone indicated that a dim probe dot had to be detected instead (Experiment 1). The probe dot could appear on the loca- tion of an old (green) element or on the location of a new (blue) element and was presented simultaneously with the new elements. The results showed that probe dot detection performance was better for probes occurring at the new locations than for those appearing at the old ones, indicating again that the observers prioritized selection of new elements. More important for the present discussion is, however, the finding that when observers had to perform a probe detection task on every trial (in their Experiment 2), the difference in probe detection performance between old and new locations was largely abandoned (for similar results, see Olivers \& Humphreys, 2002). This implies, according to Watson and Humphreys (2000), that observers actively inhibit old locations only when there is an incentive for them to do so. If there is no incentive, as in their Experiment 2, observers do not engage in the effortful process of visual marking. Accordingly, Watson and Humphreys (2000) concluded that prioritized selection of new elements is mediated by a top-down process. Even though these results show that probe detection performance is modulated by the task demands, it is questionable whether these findings necessarily indicate that prioritized selection of new over old elements is indeed mediated by a top-down process. It should be noted that in their Experiment 2, the participants were basically performing a single probe detection task in the presence of irrelevant green and blue elements. Since the probe dot was rather inconspicuous, the participants might have chosen not to divide their attention over the visual field but, instead, to limit their attentional window (see Theeuwes, 1994). Indeed, recently, Gibson and Peterson (2001) demonstrated that if participants searched for a nonsalient target, they appeared to be engaged in a focused attentional state that prevented attentional capture by a color singleton. Something similar might have occurred in Experiment 2 of Watson and Humphreys (2000). After the initial presentation of the green elements, the participants might have limited the attentional window to a small size in order to detect the nonsalient probe dot. Focusing the attentional window may have prevented the onsets accompanying the appearance of the irrelevant new elements from capturing attention, with the result that probe dot detection performance was effectively equal irrespective of the probe dot location.

In sum, studies in which the issue of whether prioritized selection is under top-down or bottom-up control has been addressed have failed to unequivocally provide evidence for one or the other view. The results of a few studies have suggested that prioritized selection is under top-down control. However, these results may also be accommodated by the alternative view. The aim of the present study was to investigate whether prioritizing multiple new elements over old elements is a bottom-up or a top-down process. To determine this, it is necessary to find out whether the new elements are prioritized under conditions in which newness is explicitly task irrelevant. As has been pointed out by Yantis and Egeth 
(1999), one can speak of selection in a purely stimulusdriven fashion only when the stimulus feature in question is completely task-irrelevant, so that there is no incentive for the observer to attend to it deliberately. As expressed by Yantis and Egeth, "If an object with such an attribute captures attention under these conditions, then and only then can that attribute be said to capture attention in a purely stimulus-driven fashion" (p. 663). For example, Jonides and Yantis (1988; see also Theeuwes, 1990; Yantis \& Jonides, 1984) used this logic when they determined whether singletons are able to capture attention in a purely stimulus-driven way. In their experiments, there was always one feature singleton present; yet the feature singleton was uncorrelated with the position of the target. In other words, there was no incentive for participants to attend deliberately to the feature singleton. Using this paradigm, Jonides and Yantis concluded that only abrupt onsets are able to capture attention exogenously in a bottom-up fashion. We used similar conditions in the present study - that is, in Experiment 1, observers searched for a target that occurred with equal probability among the old elements (through an equiluminant color change of one of the old elements at presentation of the new elements) or among the new elements. In this way, there was no incentive to deliberately attend to the new elements, for the target was equally probably present among the old and the new elements. Basically, the preview task of Watson and Humphreys (1997) was used, with the difference that the target could appear with equal probability among the old and the new elements. Since under these conditions, there is no incentive to actively inhibit the old elements, one would expect that target detection performance would be the same regardless of whether the target appears among the old or the new elements. However, if prioritizing new over old elements is an automatic bottom-up process, one would expect that target detection performance would be better when the target appeared among the new elements than when it occurred among the old elements.

\section{EXPERIMENT 1}

The aim of Experiment 1 was to investigate whether prioritized selection of new over old elements still would take place if the target occurred with equal probability among the old and the new elements. Observers had to indicate the presence or absence of a blue $H$ among green $H$ s and blue $A$ s. In the preview condition, on each trial, 10 elements consisting of green $H$ s and blue $A$ s (old elements) were concurrently presented. After $400 \mathrm{msec}$, new elements, also consisting of green $H \mathrm{~s}$ and blue $A \mathrm{~s}$, were added to the display. ${ }^{2}$ If the target occurred among the old elements, upon presentation of the new elements, one of the previously presented green $H$ s turned into blue. The color change from green to blue was not accompanied by a luminance change - that is, the green and blue colors were equiluminant to each other. If the target occurred among the new elements, one of the new elements was the blue $H$ target. The target occurred among the old elements with a probability of .25 and among the new elements with a probability of .25 , and the target was absent with a probability of .5. Performance in the preview condition was compared with that in a baseline condition in which the old and the new elements were presented all at once. If prioritization of new over old elements is under bottom-up control, it would be predicted that target detection would be faster if the target occurred among the new elements than if it occurred among the old elements. Furthermore, performance in the baseline condition should be intermediate between performances based on trials in which the target occurred among the old elements and those in which the target occurred among the new elements. Alternatively, if the observers prioritized new over old elements by the effortful application of top-down inhibition of the old elements, as was suggested by Watson and Humphreys (1997), it would be expected that performance would be equal irrespective of whether the target occurred among the old or the new elements. Furthermore, performance in the preview condition would be expected to be equal to that in the baseline condition.

\section{Method}

Participants. Five male and 7 female participants, 19 to 44 years of age, took part in the present experiment. Each participant had normal or corrected-to-normal vision.

Apparatus. A Celeron 400-MHz/128-Mb PC controlled the timing of events, the generation of the stimuli, and the recording of the responses. The stimuli were presented on a 19-in. Multiscan color monitor (with an ATI Rage 4-Mb card). The "z" key and the "/" key of the computer keyboard were utilized as response buttons. The participants were tested in a sound-isolated dimly lit room. They were seated at a distance of $95 \mathrm{~cm}$ from the computer monitor, with their heads fixed in a head- and chinrest.

Task and Stimuli. The participants had to indicate the presence or absence of a blue capital letter $H$ among green $H$ s and blue $A$ s. The target occurred on $50 \%$ of the trials. In the baseline condition, the participants were presented with 10 blue $A$ s and 10 green $H$ s in the target-absent trials and with 10 blue $A \mathrm{~s}, 9$ green $H \mathrm{~s}$, and 1 blue $H$ in the target-present trials. In the preview condition, in each trial, 5 blue $A$ s and 5 green $H$ s (old elements) were presented, followed after $400 \mathrm{msec}$ by the addition of another 10 elements (new elements). In the target-present trials, the target occurred with equal probability among the old and the new elements. If the target occurred among the old elements, one of the previously presented green $H$ s turned into blue upon the appearance of the new elements consisting of 5 blue $A$ s and 5 green $H$ s. If the target occurred among the new elements, following the presentation of the old elements, 5 blue $A \mathrm{~s}, 4$ green $H \mathrm{~s}$, and 1 blue $H$ were added. Green and blue elements (green, CIE $x, y$ chromaticity coordinates of .253 and .449 , $6.5 \mathrm{~cd} / \mathrm{m}^{2}$; blue, CIE $x, y$ chromaticity coordinates of .164 and .111 , $6.2 \mathrm{~cd} / \mathrm{m}^{2}$ ) were equiluminant as determined by a flicker fusion test (Ives, 1912) carried out at fixation. ${ }^{3}$ The background was black with a luminance of $0.0 \mathrm{~cd} / \mathrm{m}^{2}$. The letters subtended a visual angle of $0.7^{\circ} \times 0.9^{\circ}$ at an observation distance of $95 \mathrm{~cm}$ and were randomly positioned within a stimulus field of $14.9^{\circ} \times 10.7^{\circ}$ of visual angle.

Design and Procedure. A within-subjects design was used. Each participant completed one block of 64 trials corresponding to the baseline condition and one block of 128 trials corresponding to the preview condition. The order of blocks was counterbalanced 
over participants. Each block was preceded by 16 practice trials corresponding to the current condition. Target presence (target present and target absent) was varied randomly within blocks of trials. In the preview condition, target occurrence (target among the old elements and target among the new elements) was varied randomly. Each trial started with the presentation of a tone, followed immediately by the presentation of a white fixation cross in the middle of the screen. After 1,500 msec, either 20 elements were presented concurrently (baseline condition) or 10 letters (old elements) were presented for $400 \mathrm{msec}$, after which 10 letters (new elements) were added to the display (preview condition). The display remained on until the participant responded within a maximum of $6,000 \mathrm{msec}$. The fixation cross remained on throughout each trial. The participants were instructed to remain fixated until the new elements came on. Furthermore, they were told that if the target was present, it would appear upon presentation of the second set of elements. Finally, they were told that the target could occur with equal probability among the old and the new elements. Half of the participants pressed the " $\mathrm{z}$ " key when the $H$ target was present and pressed the "/" key when it was absent. This assignment was reversed for the other half of the participants.

\section{Results}

Table 1 shows the mean correct reaction times (RTs), as well as the mean percentages of errors, as a function of target presence (target present and target absent), condition (preview and baseline), and for the preview condition, target occurrence (target among old elements and target among new elements). A repeated measures analysis of variance (ANOVA) was conducted on the mean correct RTs, with target presence (target present and target absent) and condition (preview and baseline) as factors. There was a significant main effect of target presence $[F(1,11)=87.97, p<.01]$, indicating that, overall, the participants responded more quickly if the target was present than if it was absent. There was no difference between the preview and the baseline condition $[F(1,11)=$ $3.67, p>.05]$. Furthermore, there was no interaction between target presence and condition $[F(1,11)=0.61]$.

Pairwise comparisons between the different conditions for the correct RTs in the target-present trials revealed that if the target occurred among the new elements, responses were faster than if it occurred among the old elements $[F(1,11)=7.33, p<.02]$ or if it was presented in the baseline condition $[F(1,11)=7.74, p<.02]$. Furthermore, RT in the trials in which the target occurred among the old elements was equal to that in the target-present trials in the baseline condition $[F(1,11)=$ $0.01]$.
Overall, the participants made $7.9 \%$ errors. The participants more often falsely reported that the target was absent when in fact the target was present than they reported that the target was present when in fact the target was absent $[F(1,11)=46.07, p<.01]$, showing that the participants were more inclined to respond target absent than target present. Pairwise comparisons between the different conditions for the error rates in the target-present trials revealed that if the target occurred among the new elements, fewer errors were made than if the target occurred among the old elements $[F(1,11)=12.35, p<$ $.01]$. Furthermore, error rates for trials in which the target occurred among the old elements were higher than error rates in the target-present trials of the baseline condition $[F(1,11)=9.74, p<.01]$. Finally, error rates for trials in which the target occurred among the new elements were equal to those in the target-present trials of the baseline condition $[F(1,11)=0.01]$.

\section{Discussion}

Experiment 1 showed that if the target occurred among the new elements, the participants were faster than if it occurred among the old elements or if the target was presented in the baseline condition. Despite the absence of a clear incentive to attend to the new elements, the observers seemed to prioritize the selection of new elements at the expense of old elements. When applying the definition of stimulus-driven selection as put forward by Yantis and Egeth (1999), one has to conclude that new elements are selected over old elements in a bottom-up way. Indeed, other studies in which the same logic was used have concluded that onsets capture attention in a purely stimulus-driven way (e.g., Theeuwes, 1990, 1991, 1994). For example, Yantis and Jonides (1984) showed that in visual search, a target appearing with abrupt onset was more rapidly detected than a target that appeared without onset. These previous results are basically identical to those in Experiment 1, except that, in Experiment 1, multiple onsets were used instead of a single one. The implication of this is that, possibly, not only a single onset, but also multiple onsets receive attentional priority in a purely stimulus-driven manner. It is likely that the multiple abrupt onsets accompanying the presentation of the new elements generated a large bottom-up activation, with the result that new elements involuntary received attentional priority (Cave \& Wolfe,

Table 1

Mean Correct Reaction Times (RTs) and Percentages of Errors as a Function of Condition, Target Presence, and Target Occurrence in Experiment 1

Condition

\begin{tabular}{|c|c|c|c|c|c|c|}
\hline & \multicolumn{3}{|c|}{ Preview } & \multicolumn{3}{|c|}{ Baseline } \\
\hline & RT & $S D$ & $\%$ Errors & RT & $S D$ & $\%$ Errors \\
\hline Target present & 747.6 & 122.4 & 15.5 & 795.7 & 139.5 & 10.7 \\
\hline Among old elements & 792.2 & 143.9 & 20.1 & & & \\
\hline Among new elements & 704.7 & 120.9 & 10.9 & & & \\
\hline Target absent & 951.7 & 187.2 & 2.6 & $1,025.5$ & 188.2 & 0.3 \\
\hline
\end{tabular}


1990; Donk \& Theeuwes, 2001; Wolfe, Cave, \& Franzel, 1989).

Even though the results of Experiment 1 are in line with the predictions of a bottom-up account, the results of Experiment 1 do not necessarily imply that the search process through the new elements was more efficient than the search process through the old ones. Instead, it might just have been easier to detect a blue $H$ onset than a blue $H$ that appeared through an equiluminant color change. In other words, we have no evidence that the observers actually searched the new elements before searching the old ones. In Experiment 2 we used the same method as that used by Theeuwes et al. (1998) and Donk and Theeuwes (2001), in which the numbers of old and new elements were independently varied, to investigate whether the observers would completely avoid the old elements.

\section{EXPERIMENT 2}

In Experiment 2, the number of old elements and the number of new elements were independently manipulated in order to determine the search efficiency through the old and the new elements.

\section{Method}

Participants. Four male and 8 female participants, 18 to 33 years of age, took part in the present experiment. Each participant had normal or corrected-to-normal vision.

Apparatus. The apparatus was identical to that in Experiment 1.
Task and Stimuli. The task and the stimuli were identical to those in Experiment 1, except that, in the preview condition, the number of old and the number of new elements were independently manipulated. In each trial, 6,10 , or 14 letters (old elements) were presented, followed after $400 \mathrm{msec}$ by the addition of another 6,10 , or 14 letters (new elements). The baseline condition was omitted because the independent manipulation of the number of old and new elements would provide sufficient information about relative search efficiency (e.g., Donk \& Theeuwes, 2001; Theeuwes et al., 1998).

Design and Procedure. A within-subjects design was used. Each participant completed 2 blocks of 54 practice trials each, followed by 16 experimental blocks of 54 trials each (total of 864 experimental trials). Target presence (target present and target absent), the number of old elements $(6,10$, and 14), and the number of new elements $(6,10$, and 14) were varied randomly within blocks of trials. If the target was present, it could occur with equal probability among the old elements and among the new elements. The procedures were identical to those in Experiment 1.

\section{Results}

Two participants were excluded from further analyses due to error rates exceeding $10 \%$, leaving 10 participants. Figure 1 depicts the mean correct RTs as a function of target presence (target present and target absent), target occurrence (target present among the old elements and target present among the new elements), the number of old elements $(6,10$, and 14 elements), and the number of new elements $(6,10$, and 14 elements). Overall, the participants more quickly responded correctly to the presence of the target than to the absence of the target $[F(1,9)=37.55, p<.01]$.

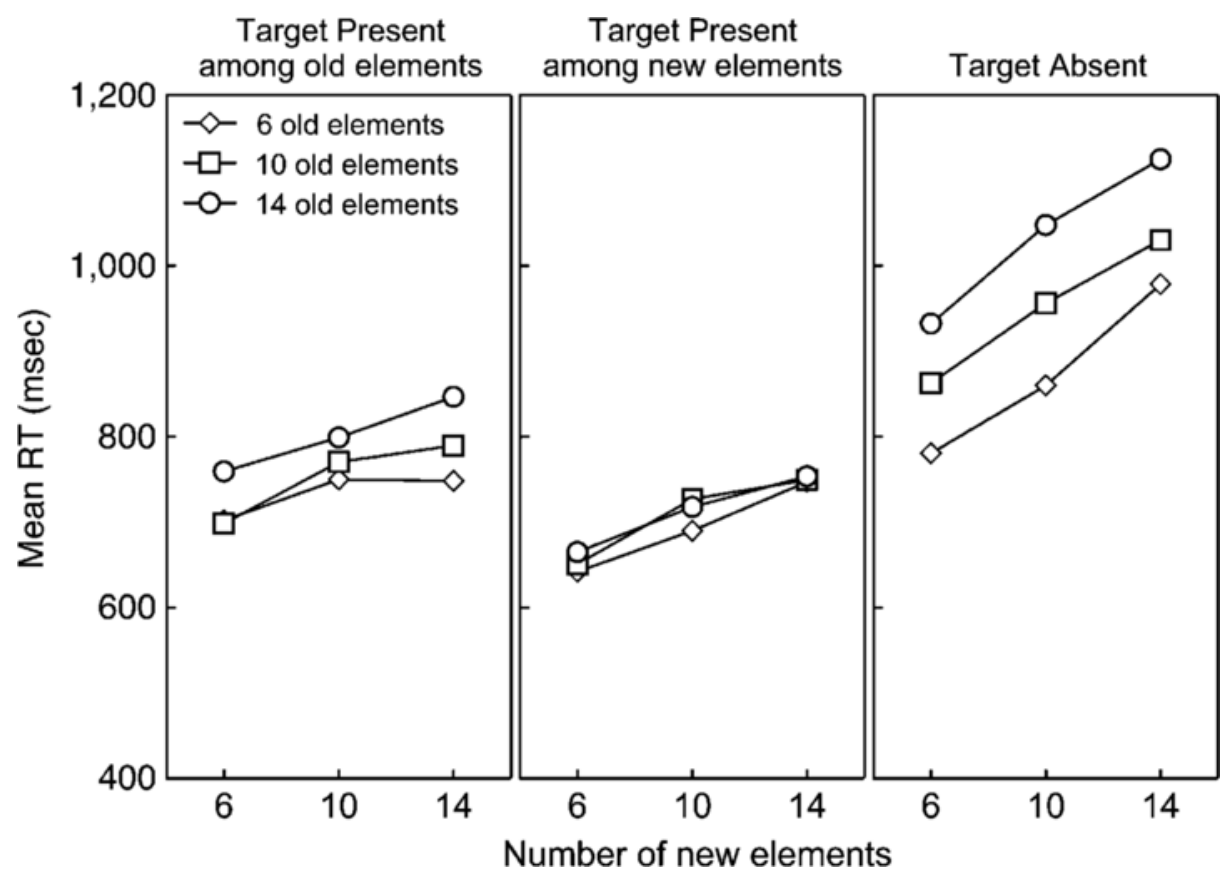

Figure 1. Correct mean reaction time (RT) as a function of the number of new elements and the number of old elements separately for trials in which the target occurs among the old elements, trials in which the target occurs among the new elements, and target-absent trials in Experiment 2. 
An ANOVA was conducted on the individual correct present-trial RTs, with target occurrence (target among the old elements and target among the new elements), the number of old elements $(6,10$, and 14 elements), and the number of new elements $(6,10$, and 14 elements) as repeated measures factors. There were statistically significant main effects of target occurrence $[F(1,9)=20.20$, $p<.01]$ and number of new elements $[F(2,18)=13.32$, $p<.01]$. Number of old elements did not affect RT $[F(2,18)=3.15, p>.05]$. The only significant interaction was between target occurrence and number of old elements $[F(2,18)=4.90, p<.02]$, implying that the number of old elements affected RT differently if the target occurred among the old elements than if it occurred among the new elements. If the target occurred among the old elements, both the number of old elements and the number of new elements affected RT [number of old elements, $F(2,18)=4.44, p<.03$; number of new elements, $F(2,18)=10.12, p<.01]$. If the target occurred among the new elements, there was only a significant effect of the number of new elements [number of old elements, $F(2,18)=1.01, p>.05$; number of new elements, $F(2,18)=10.95, p<.01]$.

To investigate whether the speed of search was equal through the old elements and the new elements, best-fitting lines were determined for the functions relating RT to the number of old elements and those relating RT to the number of new elements separately for each participant for trials in which the target occurred among the old elements, trials in which the target occurred among the new elements, and target-absent trials (see also Donk \& Theeuwes, 2001). Table 2 shows the mean search slopes and intercepts. Search slopes were larger for targetabsent trials than for target-present trials $[F(1,9)=42.48$, $p<.01]$. If the target occurred among the old elements, the slope of the function relating RT to the number of old elements was identical to the slope of the function relating RT to the number of new elements $[F(1,9)=0.06]$. If the target occurred among the new elements, the slope of the function relating RT to the number of old elements was smaller than the slope of the function relating RT to the number of new elements $[F(1,9)=14.73, p<.01]$.

The intercepts of the functions were larger for targetabsent trials than for target-present trials $[F(1,9)=10.38$, $p<.01]$. If the target occurred among the old elements, the intercept of the function relating RT to the number of old elements was identical to the intercept of the function relating RT to the number of new elements $[F(1,9)=$ 0.08 ]. If the target occurred among the new elements, the intercept of the function relating RT to the number of old elements was larger than the intercept of the function relating RT to the number of new elements $[F(1,9)=14.57$, $p<.01]$. Finally, the intercepts of the functions relating RT to the number of new elements were larger if the target occurred among the old elements than if it occurred among the new elements $[F(1,9)=40.81, p<.01]$.

Table 3 shows the mean percentages of errors. Overall, the participants made $4.36 \%$ errors. The participants more often falsely reported that the target was absent when in fact the target was present than they reported that the target was present when in fact the target was absent $[F(1,9)=36.41, p<.01]$, showing that the participants were more inclined to respond target absent than target present. Generally, error rates were either unaffected or affected in the same direction as RT.

\section{Discussion}

Experiment 2 shows that even though the target occurred with equal probability among the old and the new elements, the observers perfectly prioritized the new elements for selection over the old ones: If the target occurred among the new elements, search was completely independent of the number of old elements. In other words, the new elements were perfectly prioritized over the old elements even though there were no explicit incentives for the observers to do so.

Even though Experiment 2 clearly shows that new elements are prioritized for selection over old ones, the results are rather inconclusive regarding the exact mechanism underlying the selection process. If observers were to perform a serial search through new elements first, followed by a serial search through old elements, search slopes as a function of the number of new elements should be twice as large when the target occurred among the old elements than when it occurred among the new elements. For if the target had occurred among old elements, one would expect that, on average, twice as many new elements would have been searched through, as compared

Table 2

Mean Intercepts, Search Slopes, and Absent/Present Ratios Separately for the Functions Relating Reaction Time (RT) to the Number of Old Elements and Those Relating RT to the Number of New Elements in Experiment 2

\begin{tabular}{|c|c|c|c|c|c|c|}
\hline \multirow[b]{3}{*}{ Target Presence } & \multicolumn{6}{|c|}{ Function } \\
\hline & \multicolumn{3}{|c|}{ Old Elements } & \multicolumn{3}{|c|}{ New Elements } \\
\hline & $\begin{array}{c}\text { Intercepts } \\
(\mathrm{msec})\end{array}$ & $\begin{array}{l}\text { Slopes } \\
(\mathrm{msec})\end{array}$ & $\begin{array}{c}\text { Absent/Present } \\
\text { Ratio } \\
\end{array}$ & $\begin{array}{c}\text { Intercepts } \\
(\mathrm{msec})\end{array}$ & $\begin{array}{l}\text { Slopes } \\
(\mathrm{msec})\end{array}$ & $\begin{array}{c}\text { Absent/Present } \\
\text { Ratio } \\
\end{array}$ \\
\hline \multicolumn{7}{|l|}{ Present } \\
\hline Among old elements & 680.1 & 8.2 & 2.5 & 668.5 & 9.3 & 2.5 \\
\hline Among new elements & 680.1 & 2.4 & 8.4 & 583.4 & 12.1 & 1.9 \\
\hline Absent & 749.9 & 20.2 & & 720.2 & 23.2 & \\
\hline
\end{tabular}


Table 3

Mean Percentages of Errors in Experiment 2

\begin{tabular}{ccccc}
\hline & \multicolumn{3}{c}{ Mean Percentages of Errors in Experiment 2 } \\
\cline { 3 - 4 } Number of & & \multicolumn{2}{c}{ Target Present } & \\
Old Elements & Number of & Among & Among & Target \\
New Elements & Old Elements & New Elements & Absent \\
\hline 6 & 6 & 7.9 & 2.1 & 0.4 \\
& 10 & 7.9 & 2.9 & 1.0 \\
\multirow{2}{*}{10} & 14 & 9.2 & 7.1 & 0.8 \\
& 6 & 8.5 & 3.8 & 0.2 \\
& 10 & 13.8 & 5.0 & 1.0 \\
14 & 14 & 12.1 & 5.8 & 1.0 \\
& 6 & 15.4 & 1.3 & 1.0 \\
& 10 & 13.7 & 4.2 & 0.4 \\
& 14 & & 8.3 & 1.7 \\
\hline
\end{tabular}

with when the target occurred among new elements. Table 2 shows that this was not the case. Search slopes as a function of the number of new elements tended to be smaller if the target occurred among the old elements than if it occurred among the new elements. At this point, it is not immediately clear how to explain this finding. It should be noted, however, that this finding does not have bearing on whether prioritized selection is mediated by a top-down or a bottom-up process. Furthermore, Olivers, Humphreys, Heinke, and Cooper (2002) recently reported a similar finding. In their Experiment 1 , they had participants search for a target that occurred either among the old or among the new elements. Their major manipulation involved the probability with which the target occurred in one or the other set of elements. If the target unexpectedly occurred among the old elements, search slopes were surprisingly low. In fact, search was then as efficient as when the target occurred among the new elements. The present results are in line with these earlier findings. Possibly, observers always serially deploy attention to each of the new element locations until the target is found. If the target is not found, they may redeploy attention again to a number of new elements before turning to the old elements. The tendency to redeploy attention to the new elements might be related to the relatively high strength of bottom-up activation of the new elements, as compared with the old elements, due to the recent onset of the new elements. As time passes, the strength of bottom-up activation for the new elements declines and starts to equal that for the old elements, resulting in increasingly more old elements being selected for attention. Consequently, if the number of new elements is low, attention may be repeatedly deployed to new elements before old elements are selected. If the number of new elements is high, attention might be serially deployed to new elements only once before old elements are inspected. In the latter case, the relatively higher activation of the new elements might be diminished at the time all the new elements are inspected, resulting in only a single serial scan of the new elements. Obviously, such an explanation is rather speculative and requires further research.
The finding of perfect prioritization of new over old elements in the present experiment suggests that the underlying mechanism operates in a bottom-up fashion. Nevertheless, one may argue that even though the observers had no incentive to prioritize new over old elements in Experiment 2, the application of a voluntary strategy to attend to onsets would not be harmful either. It is theoretically possible that the observers were intentionally set to attend to onsets because there was little reason to attend to any other property. Indeed, Folk, Remington, and Johnston (1992) have claimed that observers may voluntarily use certain salient features such as onsets to guide focal attention in the search process, as long as it is not harmful for them to do so: If "there is little motivation to configure the system for any other property, abrupt luminance change (or dynamic discontinuities in general) may be instantiated as the 'default' setting" (Folk et al., 1992, p. 1042). It is possible that the results of Experiment 2 are due to the application of such a voluntary top-down configuration. Although such a top-down setting would not have been directly profitable, neither might it have been disadvantageous. The aim of Experiment 3 was to investigate this possibility by increasing the likelihood that the target would occur among old elements.

\section{EXPERIMENT 3}

In Experiment 3, the number of old elements and the number of new elements were, as in Experiment 2, independently manipulated to determine search efficiency through the old and the new elements. In addition, if present, the target occurred twice as often among the old elements than among the new elements. Accordingly, applying any top-down configuration in order to prioritize onsets for selection would be disadvantageous in the majority of trials.

\section{Method}

Participants. Five male and 3 female participants, 19 to 30 years of age, took part in the present experiment. Each participant had normal or corrected-to-normal vision. 
Apparatus. The apparatus was identical to that in Experiment 2.

Task and Stimuli. The task and stimuli were identical to those in Experiment 2, except that the target occurred twice as often among the old elements than among the new elements.

Design and Procedure. A within-subjects design was used. Each participant completed 1 block of 54 practice trials, followed by 24 experimental blocks of 54 trials each (total of 1,296 experimental trials). Target presence (target present and target absent), the number of old elements $(6,10$, and 14), and the number of new elements $(6,10$, and 14) were varied randomly within blocks of trials. If the target was present, it occurred twice as often among the old elements than among the new elements. This resulted in 432 trials in which the target occurred among the old elements, 216 trials in which the target occurred among the new elements, and 648 trials in which the target was absent. The participants were explicitly informed about the likelihood with which the target could occur among the old and the new elements and were told to use this information. The procedures were otherwise identical to those in Experiment 2 .

\section{Results}

Figure 2 depicts the mean correct RTs as a function of target presence (target present and target absent), target occurrence (target present among the old elements and target present among the new elements), the number of old elements $(6,10$, and 14 elements), and the number of new elements $(6,10$, and 14 elements). Overall, the participants responded correctly more quickly to the presence of the target than to the absence of the target $[F(1,7)=40.58, p<.01]$.

An ANOVA was conducted on the individual correct present-trial RTs, with target occurrence (target among the old elements and target among the new elements), the number of old elements $(6,10$, and 14 elements), and the number of new elements $(6,10$, and 14 elements) as repeated measures factors. There were statistically significant main effects of target occurrence $[F(1,7)=$ $19.37, p<.01]$, number of old elements $[F(2,14)=7.33$, $p<.01]$, and number of new elements $[F(2,14)=29.81$, $p<.01]$. The only significant interaction was between target occurrence and number of old elements $[F(2,14)=$ $8.69, p<.01]$, implying that the number of old elements affected RT differently when the target occurred among the old elements than when it occurred among the new elements. If the target occurred among the old elements, both the number of old elements and the number of new elements affected RT [number of old elements, $F(2,14)=$ $29.54, p<.01$; number of new elements, $F(2,14)=3.96$, $p<.04]$. If the target occurred among the new elements, there was only a significant effect of the number of new elements [number of old elements, $F(2,14)=0.42$; number of new elements, $F(2,14)=20.13, p<.01]$.

To investigate whether the speed of search was equal through the old elements and the new elements, best-fitting lines were determined for the functions relating RT to the number of old elements and those relating RT to the number of new elements separately for each participant for trials in which the target occurred among the old elements, trials in which the target occurred among the new elements, and target-absent trials (see also Donk \& Theeuwes, 2001). Table 4 shows the mean search slopes and intercepts. Search slopes were larger for target-

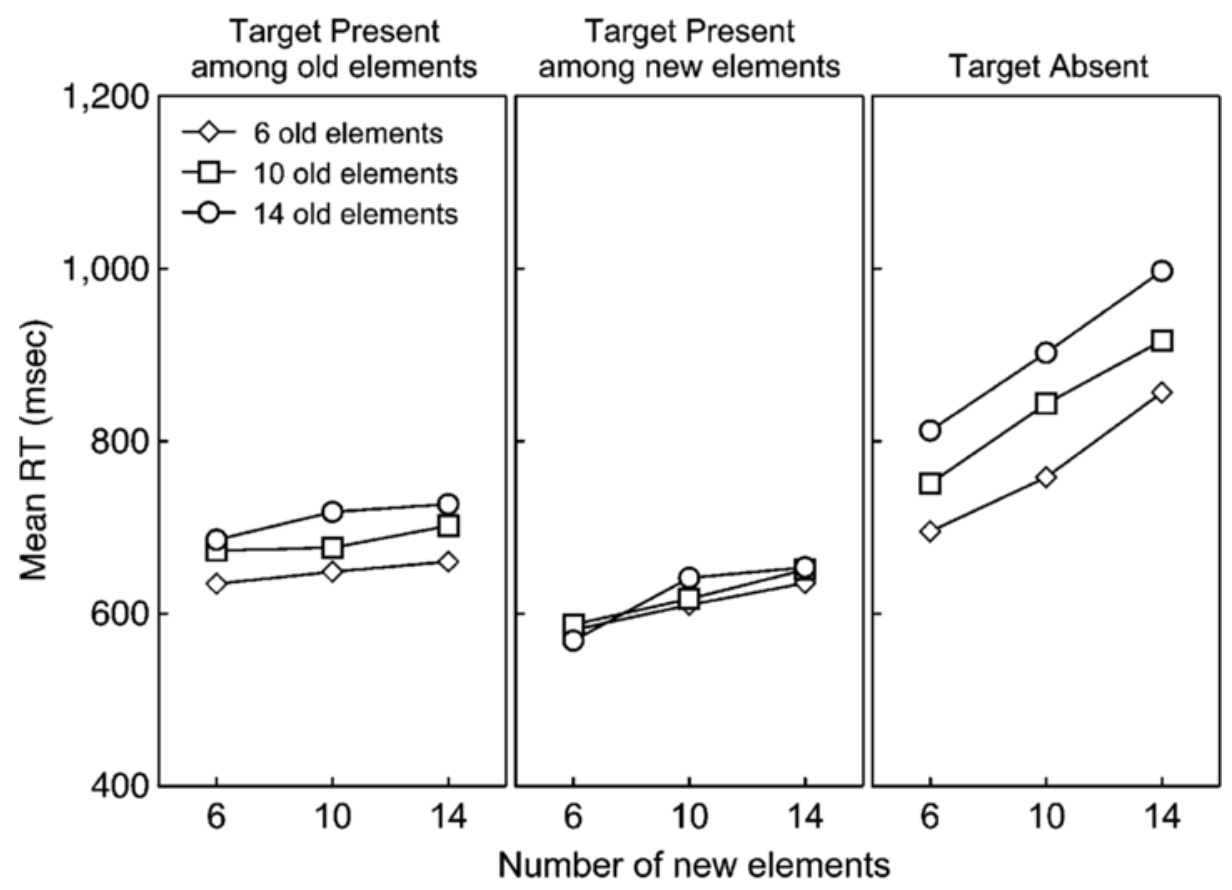

Figure 2. Correct mean reaction time (RT) as a function of the number of new elements and the number of old elements separately for trials in which the target occurs among the old elements, trials in which the target occurs among the new elements, and target-absent trials in Experiment 3. 
Table 4

Mean Intercepts, Search Slopes, and Absent/Present Ratios Separately for the Functions Relating Reaction Time (RT) to the Number of Old Elements and Those Relating RT to the Number of New Elements in Experiment 3

\begin{tabular}{|c|c|c|c|c|c|c|}
\hline \multirow[b]{3}{*}{ Target Presence } & \multicolumn{6}{|c|}{ Function } \\
\hline & \multicolumn{3}{|c|}{ Old Elements } & \multicolumn{3}{|c|}{ New Elements } \\
\hline & $\begin{array}{l}\text { Intercepts } \\
(\mathrm{msec})\end{array}$ & $\begin{array}{l}\text { Slopes } \\
(\mathrm{msec})\end{array}$ & $\begin{array}{c}\text { Absent/Present } \\
\text { Ratio }\end{array}$ & $\begin{array}{c}\text { Intercepts } \\
(\mathrm{msec})\end{array}$ & $\begin{array}{l}\text { Slopes } \\
(\mathrm{msec})\end{array}$ & $\begin{array}{c}\text { Absent/Present } \\
\text { Ratio } \\
\end{array}$ \\
\hline \multicolumn{7}{|l|}{ Present } \\
\hline Among old elements & 602.2 & 7.8 & 2.1 & 640.4 & 4.0 & 5.3 \\
\hline Among new elements & 601.1 & 1.5 & 11.1 & 531.6 & 8.5 & 2.5 \\
\hline Absent & 669.4 & 16.7 & & 624.0 & 21.3 & \\
\hline
\end{tabular}

absent trials than for target-present trials $[F(1,7)=24.53$, $p<.01]$. If the target occurred among the old elements, the slope of the function relating RT to the number of old elements was equal to those relating RT to the number of new elements $[F(1,7)=5.17, p>.05]$. If the target occurred among the new elements, the slope of the function relating RT to the number of old elements was smaller than the slopes of the functions relating RT to the number of new elements $[F(1,7)=7.47, p<.03]$.

The intercepts of the functions were larger for targetabsent trials than for target-present trials $[F(1,7)=21.86$, $p<.01]$. If the target occurred among the old elements, the intercept of the function relating RT to the number of old elements was identical to the intercept of the function relating RT to the number of new elements $[F(1,7)=$ $4.92, p>.05]$. If the target occurred among the new elements, the intercept of the function relating RT to the number of old elements was larger than the intercept of the function relating $\mathrm{RT}$ to the number of new elements $[F(1,7)=7.50, p<.03]$. Finally, the intercept of the functions relating RT to the number of new elements was larger if the target occurred among the old elements than if it occurred among the new elements $[F(1,7)=10.18$, $p<.02]$.

Table 5 shows the mean percentages of errors. Overall, the participants made $3.3 \%$ errors. The participants more often falsely reported the target to be absent when in fact the target was present than the target to be present when in fact the target was absent $[F(1,7)=10.12$, $p<.02]$, showing that the participants were more in- clined to respond target absent than target present. Generally, error rates were either unaffected or affected in the same direction as RT.

\section{Discussion}

The results of Experiment 3 basically replicated those obtained earlier in Experiment 2. New elements were perfectly prioritized for selection over old elements even though the observers knew that the probability with which the target occurred among the old elements was twice as large as the probability with which it occurred among the new elements. Despite the presence of a strong incentive not to prioritize the new elements, the observers appeared to be unable to do so. These results suggest that prioritized selection of new elements is the result of a bottom-up process.

That in Experiment 3, as in Experiment 2, search slopes as a function of the number of new elements tended to be smaller if the target occurred among the old elements than if it occurred among the new elements is peculiar. As was remarked before, it appears as if observers perform repeated serial searches through the new elements before the old elements are inspected. The tendency to repeatedly deploy attention to new elements might depend on a stronger bottom-up activation of the new elements, as compared with the old elements, caused by their relative recent onset. Owing to a decline in the activation of the new elements over time, old elements may start to "leak through" the attentional selection system, resulting in an increasing probability of

Table 5

Mean Percentages of Errors in Experiment 3

\begin{tabular}{ccccc}
\hline \multirow{2}{*}{$\begin{array}{c}\text { Number of } \\
\text { Old Elements }\end{array}$} & $\begin{array}{c}\text { Number of } \\
\text { New Elements }\end{array}$ & $\begin{array}{c}\text { Among } \\
\text { Old Elements }\end{array}$ & $\begin{array}{c}\text { Among } \\
\text { New Elements }\end{array}$ & $\begin{array}{c}\text { Target } \\
\text { Absent }\end{array}$ \\
\hline 6 & 6 & 8.9 & 1.6 & 1.9 \\
& 10 & 3.4 & 2.1 & 1.7 \\
\multirow{2}{*}{10} & 14 & 5.7 & 3.1 & 2.4 \\
& 6 & 9.1 & 1.6 & 1.6 \\
& 10 & 6.3 & 2.6 & 1.9 \\
14 & 14 & 8.3 & 2.1 & 2.6 \\
& 6 & 11.7 & 0.5 & 1.6 \\
& 10 & 12.0 & 3.1 & 1.4 \\
& 14 & 9.4 & 3.7 & 1.6 \\
\hline
\end{tabular}


their selection as time passes. If this is true, search slopes of functions relating RT to the number of new elements can become more flat if the target occurs among old elements than if it occurs among new elements. In addition, intercepts of these functions should then be larger in the former than in the latter case. The results show that this is indeed the case.

Regardless of this speculative explanation of the search slopes when the target occurs among the old elements, the absence of any effect of the number of old elements when the target occurs among the new elements shows that prioritization was perfect.

\section{GENERAL DISCUSSION}

The experiments in the present study demonstrate that prioritized selection of new elements over old ones occurs even in the absence of any incentive to do so. Despite the fact that, in both Experiments 1 and 2, target occurrence was uncorrelated with newness and the participants were explicitly informed about this, search efficiency was not affected by the number of old elements when the target occurred among the new ones. Even in Experiment 3, in which the participants knew that the target would occur twice as often among the old than among the new elements, perfect prioritized selection for new elements was obtained.

The present results are at odds with a view that assumes that prioritized selection of new elements is the result of a top-down-operating inhibition mechanism (Olivers \& Humphreys, 2002; Watson \& Humphreys, 1997, 2000). In fact, within the visual-marking account, it is generally assumed that observers voluntarily and effortfully prioritize new elements for selection only if it is beneficial for performance to do so. For example, Watson and Humphreys (2000) concluded, on the basis of their results, that "old stimuli could be inhibited but only when there was an advantage for subjects to ignore the old stimuli .... When there was no advantage to be gained ...., inhibition appeared not to be applied" (p. 481). In a similar vein, Olivers and Humphreys (2002) claimed that "observers inhibit old information only when it helps to perform the task" (p. 33). The present data clearly show that prioritized selection of new elements also occurs if there is no incentive for observers. In fact, it occurs even when it is disadvantageous to do so. This suggests that prioritized selection of new elements does not have to be mediated by effortful topdown controlled processing but can be stimulus driven. ${ }^{4}$

Indeed, as was outlined in the introduction, the results of previous experiments in which the issue of attentional control has been addressed (i.e., Olivers \& Humphreys, 2002; Watson \& Humphreys, 1997, 2000) may also be accounted for by a bottom-up view. Earlier failures to find evidence for bottom-up control in prioritized selection of new elements might have been related to observers' being engaged in a focused attentional state to perform a concurrent central load task (Watson \& Humphreys, 1997, Experiment 8) or to detect a dim probe dot (Olivers \& Humphreys, 2002; Watson \& Humphreys, 2000). When observers narrow down their attentional window, attentional capture by singleton features is prevented (e.g., Joseph, Chun, \& Nakayama, 1997; Theeuwes, 1991; Yantis \& Jonides, 1990). In the present study, the participants likely divided attention over the visual field so as to allow target detection. As a result, the sudden onsets accompanying the appearance of the new elements may have acted as peripheral cues summoning attention to their locations automatically (see also Donk \& Theeuwes, 2001). ${ }^{5}$

The idea that observers may prioritize multiple onsets for selection is not new. However, previous studies (Yantis \& Johnson, 1990; Yantis \& Jones, 1991) on multiple onsets suggest that there is an upper limit of four to the maximum possible number of onsets that may receive priority. The present results show that observers may prioritize up to 14 new elements for selection. A possible explanation for this discrepancy may be related to the fact that, in previous studies, luminance onsets were always accompanied by luminance offsets, whereas this was not the case in the present study. The offsets, like the onsets, may have received prioritized selection (Theeuwes, 1991), suggesting the existence of some upper limit to the maximum number of onsets that can be prioritized (see also Donk \& Theeuwes, 2001). The present study shows that, if present at all, such an upper limit might be much higher than has previously been suggested.

The present findings show not only that new elements are prioritized over old ones, but also that old elements apparently cannot be prioritized. The results of Experiment 3 show that if the target occurred among the old elements, search efficiency was strongly dependent on the number of new elements. These results are inconsistent with the ideas put forward by Jiang, Chun, and Marks (2002), who proposed that the preview effect might not be based on inhibitory filtering, as had been proposed by Watson and Humphreys (1997), but merely on the ability of observers to group subsets of elements if there is a temporal separation between their onset. Accordingly, Jiang et al. assumed that there is no intrinsic advantage of new over old elements, because observers are presumed to be able to selectively allocate attention to the relevant subset of elements - that is, the subset of elements most likely containing the target. To provide evidence for their view, they performed an experiment (Experiment 4) in which participants had to indicate whether the rotation of a $\operatorname{target} T$ was up, down, left, or right. The target was always presented among old elements containing multiple L-shaped objects. New elements consisting of multiple rotated $T \mathrm{~s}$ were added to the display after $150 \mathrm{msec}$. In one condition, the valid preview condition, the previewed elements maintained their location upon presentation of the new elements, whereas in another condition, the invalid preview condition, the previewed elements moved at random to previously unoccupied locations as the new elements were added. Previous studies had shown that performance in the invalid preview condition was similar to that in a standard conjunction 
condition. As such, it provided the baseline for assessing the extent of the preview effect. The results indicated that the accuracy in reporting the orientation of the old $T$ was better in the valid than in the invalid preview condition. Jiang et al. concluded that the participants were able to prioritize old over new elements for selection. However, it should be noted that performance in the valid preview condition was far from perfect. If no new elements had been added to the display, it is likely that the accuracy in reporting the orientation of the unique $T$ among the old elements would have been close to $100 \%$. Nevertheless, performance in the valid preview condition lingered around $65 \%$ accuracy, suggesting that, if anything, prioritizing was not complete at all. Therefore, in line with the present results, it seems more appropriate to assume that only new elements can be prioritized for selection.

In our experiments, we showed that prioritized selection of new over old elements could be obtained without any incentive. We cannot rule out the possibility that, in other experiments, observers may also voluntarily inhibit old elements. However, the results of both Experiments 2 and 3 demonstrate that without any incentive, perfect prioritization is obtained (i.e., there is absolutely no effect of the number of old elements). This renders an inhibition account at least questionable. In other words, if prioritization is already perfect on the basis of onset capture, why do we need an additional mechanism that assumes top-down inhibition? It should be noted that our task (search for a blue $H$ among green $H$ s and blue $A$ s) was similar to that used in most of the previous studies on the preview benefit. Therefore, this makes it highly unlikely that inhibition occurred in these previous experiments.

In sum, the present results provide strong evidence against the original visual-marking account, as proposed by Watson and Humphreys (1997). The idea that prioritized selection of new over old elements is mediated by the effortful application of inhibition to the locations of the old elements is incompatible with the present findings. The most parsimonious account is to assume that prioritized selection of new elements is mediated by a bottom-up process. It seems as if the abrupt onsets accompanying the appearance of the new elements generate a large bottom-up activation, biasing observers to prioritize the processing of new elements over old ones.

\section{REFERENCES}

Allport, D. A. (1987). Selection for action: Some behavioral and neurophysiological considerations of attention and action. In H. Heuer \& A. F. Sanders (Eds.), Perspectives on perception and action (pp. 395419). Hillsdale, NJ: Erlbaum.

Broadbent, D. E. (1958). Perception and communication. New York: Pergamon.

CAve, K. R., \& Wolfe, J. M. (1990). Modeling the role of parallel processing in visual search. Cognitive Psychology, 22, 225-271.

Donk, M., \& Theeuwes, J. (2001). Visual marking beside the mark: Prioritizing selection by abrupt onsets. Perception \& Psychophysics, 63, 891-900.

Folk, C. L., Remington, R. W., \& Johnston, J. C. (1992). Involuntary covert orienting is contingent on attentional control settings. Journal of Experimental Psychology: Human Perception \& Performance, 18, 1030-1044.

Gibson, B. S., \& Peterson, M. A. (2001). Inattentional blindness and attentional capture: Evidence for attention-based theories of visual salience. In C. L. Folk \& B. S. Gibson (Eds.), Attraction, distraction and action: Multiple perspectives on attentional capture (pp. 51-76). Amsterdam: Elsevier.

Humphreys, G. W., Watson, D. G., \& Joliceeur, P. (2002). Fractionating the preview benefit in search: Dual-task decomposition of visual marking by timing and modality. Journal of Experimental Psychology: Human Perception \& Performance, 28, 640-660.

IvES, H. E. (1912). Studies of the photometry of different colours: I. Spectral luminosity curves obtained by equality of brightness photometer and the flicker photometer under similar conditions. Philosophical Magazine, 24, 149-188.

Jiang, Y., Chun, M. M., \& Marks, L. E. (2002). Visual marking: Selective attention to asynchronous temporal groups. Journal of Experimental Psychology: Human Perception \& Performance, 28, 717 730.

Jonides, J., \& YANTIS, S. (1988). Uniqueness of abrupt visual onset in capturing attention. Perception \& Psychophysics, 43, 346-354.

Joseph, J. S., Chun, M. M., \& Nakayama, K. (1997). Attentional requirements in a "preattentive" feature search task. Nature, 387, 805807.

Olivers, C. N. L., \& Humphreys, G. W. (2002). When visual marking meets the attentional blink: More evidence for top-down, limitedcapacity inhibition. Journal of Experimental Psychology: Human Perception \& Performance, 28, 22-42.

Olivers, C. N. L., Humphreys, G. W., Heinke, D., \& Cooper, A. C. G. (2002). Prioritization in visual search: Visual marking is not dependent on a mnemonic search. Perception \& Psychophysics, 64, 540-560.

Spence, C., \& Driver, J. (1997). Audiovisual links in exogenous covert spatial orienting. Perception \& Psychophysics, 59, 1-22.

Theeuwes, J. (1990). Perceptual selectivity is task-dependent: Evidence from selective search. Acta Psychologica, 74, 81-99.

Theeuwes, J. (1991). Exogenous and endogenous control of attention: The effect of visual onsets and offsets. Perception \& Psychophysics, 49, 83-90.

Theeuwes, J. (1994). Stimulus-driven capture and attentional set: Selective search for color and visual abrupt onsets. Journal of Experimental Psychology: Human Perception \& Performance, 20, 799-806.

Theeuwes, J., Kramer, A. F., \& Atchley, P. (1998). Visual marking of old objects. Psychonomic Bulletin \& Review, 5, 130-134.

Watson, D. G., \& Humphreys, G. W. (1997). Visual marking: Prioritizing selection for new objects by top-down attentional inhibition of old objects. Psychological Review, 104, 90-122.

Watson, D. G., \& Humphreys, G. W. (2000). Visual marking: Evidence for inhibition using a probe-dot detection paradigm. Perception \& Psychophysics, 62, 471-481.

Wolfe, J. M., Cave, K. R., \& Franzel, S. L. (1989). Guided Search: An alternative to the feature integration model for visual search. Journal of Experimental Psychology: Human Perception \& Performance, 15, 419-433.

YANTIS, S., \& EgETh, H. E. (1999). On the distinction between visual salience and stimulus-driven attentional capture. Journal of Experimental Psychology: Human Perception \& Performance, 25, 661-676.

Yantis, S., \& Hillst rom, A. P. (1994). Stimulus-driven attentional capture: Evidence from equiluminant visual objects. Journal of Experimental Psychology: Human Perception \& Performance, 20, 95-107.

YANTIS, S., \& Johnson, D. N. (1990). Mechanisms of attentional priority. Journal of Experimental Psychology: Human Perception \& Performance, 16, 812-825.

YANTIS, S., \& JonES, E. (1991). Mechanisms of attentional selection: Temporally modulated priority tags. Perception \& Psychophysics, 50, 166-178.

YANTIS, S., \& JoNidES, J. (1984). Abrupt visual onsets and selective attention: Evidence from visual search. Journal of Experimental Psychology: Human Perception \& Performance, 10, 601-621.

YANTIS, S., \& JoNidES, J. (1990). Abrupt visual onsets and selective attention: Voluntary versus automatic allocation. Journal of Experimental Psychology: Human Perception \& Performance, 16, 121-134. 


\section{NOTES}

1. We use the term onset to refer to the presentation of a stimulus accompanied by a luminance increment.

2. According to Watson and Humphreys (1997), a separation of $400 \mathrm{msec}$ between the presentations of the old and the new elements is sufficient to prioritize selection of new elements in the preview paradigm.

3. Owing to retinal inhomogeneities, deviance from isoluminance may occur in the periphery. Such an effect would go against a hypothesis that states that prioritized selection of new elements is based on bottom-up control.

4. Alternatively, one may argue that it may be efficient to prioritize the set of elements that has the easiest-to-find target. Since targets occurring among new elements were always abrupt onsets (and targets occurring among old elements were not), it may have been more efficient to voluntarily prioritize new over old elements (as was suggested by one of the reviewers). Even though this is a valid argument, a view assuming observers to voluntarily prioritize elements that are most easy to find becomes effectively indistinguishable from a bottom-up view. Both accounts would predict that prioritization is dependent on saliency.

5. Recently, Humphreys, Watson, and Jolicœur (2002) showed that not only the presence of a concurrent visual task, but also the presence of a simultaneous auditory task during the preview disrupts the observer's ability to prioritize new over old elements. However, the auditory task was shown to disrupt performance only if it was presented simultaneously with the beginning of the preview period. If it was presented after the onset of the old elements but before the presentation of the new ones, it was not disruptive. In view of the present findings, it is not immediately clear why an auditory secondary task would disrupt performance if it is presented concurrently with the onset of the old elements. It is feasible that some adverse auditory-visual interaction may have altered the attentional set so that visual attention was captured by the location of the auditory stimulus (see Spence \& Driver, 1997).

(Manuscript received July 9, 2001; revision accepted for publication May 8, 2003.) 